\title{
Regge limit of gauge theory amplitudes beyond leading power approximation
}

\author{
Alexander Penin \\ Institute for Theoretical Physics, ETH Zürich, \\ Zürich 8093, Switzerland \\ Department of Physics, University of Alberta, \\ Edmonton, AB T6G 2J1, Canada \\ E-mail: penin@phys.ethz.ch
}

ABSTRACT: We study the high-energy small-angle Regge limit of the fermion-antifermion scattering in gauge theories and consider the part of the amplitude suppressed by a power of the scattering angle. For abelian gauge group all-order resummation of the doublelogarithmic radiative corrections to the leading power-suppressed term is performed. We find that when the logarithm of the scattering angle is comparable to the inverse gauge coupling constant the asymptotic double-logarithmic enhancement overcomes the power suppression, a formally subleading term becomes dominant, and the small-angle expansion breaks down. In QCD we show that in the color-singlet channel for sufficiently small scattering angles the abelian power-suppressed contribution becomes comparable to the one of BFKL pomeron. Possible role of the subleading-power effects for the solution of the unitarity problem of perturbative Regge analysis in QED and QCD is discussed. An intriguing relation between the asymptotic behavior of the power-suppressed amplitudes in Regge and Sudakov limits is discovered.

KeYwords: Effective Field Theories, Perturbative QCD, Resummation, Scattering Amplitudes

ArXiv EPrint: 1909.02013 


\section{Contents}

1 Introduction 1

2 Small-angle expansion of the Born amplitude 2

3 One-loop logarithmic corrections 3

4 Resummation of subleading-power double logarithms 5

\section{Introduction}

Small-angle or Regge limit of high-energy scattering describes a kinematical configuration with vanishing ratio of the characteristic momentum transfer to the total energy of the process. The asymptotic behavior of the gauge theory amplitudes in Regge limit remains in the focus of the theoretical studies since the early days of QED and QCD [1-7]. Despite a crucial simplification due to decoupling of the light-cone and transversal degrees of freedom, the gauge interactions in this limit possess highly nontrivial dynamics giving a rigorous quantum field theory realization of Regge concept for high-energy scattering [8]. Major progress has been achieved in the analysis of the leading-power amplitudes which scale at small momentum transfer as a ratio of the Mandelstam variables $s / t \sim 1 / \theta^{2}$, where $\theta$ is the scattering angle. It culminated in the evaluation of the next-to-leading QCD corrections to the theory of BFKL pomeron [9]. At the same time very little is known about the asymptotic behavior of the amplitudes suppressed at high energy by a power of an infrared scale. In Regge limit the power corrections to the scattering amplitudes in the impact parameter and mass have been considered in refs. [10] and [11]. In this paper we focus on the asymptotic behavior of the contributions suppressed by a small ratio $\tau=|t / s|$. In contrast to the one of the leading-power amplitudes it is determined by the double-logarithmic radiative corrections which include the second power of the large logarithm $\ln \tau$ per each power of the gauge coupling constant $\alpha$. This type of correction has been discussed so far in QED only for the amplitudes which do not have the leading-power contribution and remain finite in the small-angle limit, such as the electron-to-muon pair forward annihilation [12]. ${ }^{1}$ However for the general scattering case the logarithmically-enhanced power-suppressed contributions can also play a crucial role. Indeed a recent study of the mass-suppressed amplitudes in the high-energy fixed-angle Sudakov limit [14-18] revealed that for some processes the double-logarithmic corrections result in strong enhancement of the power-suppressed terms which asymptotically makes them comparable to the leading-power contributions, i.e. formally lead to breakdown of

\footnotetext{
${ }^{1}$ The power-suppressed logarithmically-enhanced corrections have been discussed also for EinsteinHilbert gravity and supergravity in [13].
} 
the small-mass expansion. If a similar scenario is realised in the small-angle scattering, it may significantly alter our understanding of the dynamics of gauge theory in the Regge limit. Thus a systematic renormalization group analysis of subleading-power amplitudes is of primary theoretical interest.

In this paper we make the first step toward this goal and discuss the double-logarithmic behaviour of the leading power-suppressed contribution to the fermion-antifermion scattering amplitude. First we consider an abelian gauge theory and set up an effective theory framework for the analysis of the Born scattering in Regge limit. We apply it to the calculation of the one-loop power-suppressed double-logarithmic contribution and then perform the resummation of the double-logarithmic corrections to all orders of perturbation theory to find the asymptotic behavior of the amplitude. Finally we discuss the qualitative features and the impact of the power-suppressed terms in the theory of Regge limit in QED and QCD.

\section{Small-angle expansion of the Born amplitude}

We start with the scattering of a fermion with initial momentum $p$ and final momentum $p^{\prime}=p+q$ by an external abelian field $A_{\mu}$ in the limit $q \rightarrow 0$. We choose the reference frame in a such way that the incoming fermion momentum $p^{\mu}=(\varepsilon,-\varepsilon, 0,0)$ has only one light-cone component $p^{-}=p_{+}=\sqrt{2} \varepsilon$, while $p^{+}=p_{-}=0$. In the high-energy limit one can neglect the fermion mass and the on-shell conditions $p^{2}={p^{\prime}}^{2}=0$ imply the following scaling of the momentum transfer components $q_{ \pm}=\mathcal{O}(\tau), q_{\perp}=\mathcal{O}\left(\tau^{1 / 2}\right)$, with $\tau=-q^{2} /(2 \varepsilon)^{2}$ and $q^{2}=2 q_{+} q_{-}-q_{\perp}^{2}=t$. By expanding a solution of the free Dirac equation $\psi\left(p^{\prime}\right)$ in $q$ we obtain the following series for the scattering amplitude

$$
A^{\mu} j_{\mu}=A_{-} j_{+}+\frac{\tilde{F}_{+-}}{4 p_{+}} j_{+}^{5}+\frac{i F_{+-}}{4 p_{+}} j_{+}+\mathcal{O}\left(\tau^{3 / 2}\right)
$$

where the currents read

$$
\begin{aligned}
j_{\mu} & =g \bar{\psi}\left(p^{\prime}\right) \gamma_{\mu} \psi(p), \\
j_{+} & =g \bar{\psi}(p) \gamma_{+} \psi(p), \\
j_{+}^{5} & =g \bar{\psi}(p) \gamma_{+} \gamma_{5} \psi(p),
\end{aligned}
$$

and $g$ is the fermion charge. In eq. (2.1) $F_{\mu \nu}$ and $\tilde{F}_{\mu \nu}$ are the gauge field strength tensor and its dual, respectively. They correspond to the electric and magnetic fields aligned with the direction of the initial fermion motion. From the scaling of the momentum $q$ components we find $\tilde{F}_{+-}=\mathcal{O}\left(\tau^{1 / 2}\right)$ and $F_{+-}=\mathcal{O}(\tau)$. Note that the power suppressed terms in eq. (2.1) are quite similar to the $\mathcal{O}(v)$ Pauli and $\mathcal{O}\left(v^{2}\right)$ Darwin interaction of a nonrelativistic massive fermion to the magnetic and electric fields resulting from the expansion of the Dirac Lagrangian in small fermion velocity $v$. We can now apply eq. (2.1) for the analysis of the fermion-antifermion scattering

$$
\psi\left(p_{1}\right)+\bar{\psi}\left(p_{2}\right) \rightarrow \psi\left(p_{4}\right)+\bar{\psi}\left(p_{3}\right)
$$




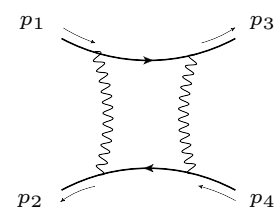

(a)

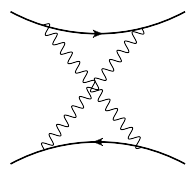

(b)

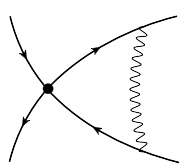

(c)

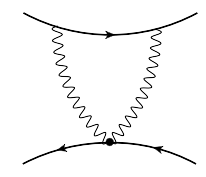

(d)

Figure 1. (a) planar and (b) nonplanar one-loop box diagrams giving rise to the leadingpower Glauber phase; (c) an effective theory diagram giving rise to the power-suppressed doublelogarithmic contribution; (d) a seagull effective theory diagram. The black dot corresponds to the effective contact $\mathcal{O}(\tau)$ interaction in eq. (2.4) and the symmetric diagram is not shown.

with the initial momenta $p_{1}^{\mu}=(\varepsilon,-\varepsilon, 0,0)$ and $p_{2}^{\mu}=-(\varepsilon, \varepsilon, 0,0)$. The corresponding Born amplitude reads

$$
\mathcal{M}^{(0)}=\mathcal{M}^{v}+\frac{\tau}{2}\left(\mathcal{M}^{v}+\mathcal{M}^{a}\right)+\mathcal{O}\left(\tau^{2}\right)
$$

where the vector and axial component are defined as follows

$$
t \mathcal{M}^{v}=j_{+}\left(p_{1}\right) j_{-}\left(p_{2}\right), \quad t \mathcal{M}^{a}=j_{+}^{5}\left(p_{1}\right) j_{-}^{5}\left(p_{2}\right) .
$$

In close analogy with the nonrelativistic expansion, the leading order amplitude is mediated by a static potential ${ }^{2}$ and the power corrections describe a contact fermion-antifermion interaction. Note that if we perform the expansion in the $u$-channel rather than in the $s$-channel i.e. about the momentum $p_{3}$ instead of $p_{2}$, the sign of the axial term in eq. (2.4) changes.

\section{One-loop logarithmic corrections}

Let us now discuss the one-loop amplitude. The corrections enhanced by a power of $\ln \tau$ can only be produced by the one-particle irreducible box diagrams in figure 1(a), 1(b). Hence, we do not consider the factorizable correction where a gauge boson is emitted and absorbed by the same fermion line. The double-logarithmic contribution comes from the virtual momentum region where $l \ll \sqrt{s}$ and the virtual fermion or antifermion is close to its mass-shell [20]. Thus we can use the same scaling rules for the components of $l$ as for the components of the momentum transfer $q$. Then it is sufficient to consider the eikonal approximation for the (anti)fermion propagator

$$
S\left(l-p_{1,2}\right) \rightarrow \frac{\gamma_{\mp}}{2 l_{\mp} \pm l_{\perp}^{2} / \sqrt{s / 2} \pm i \epsilon},
$$

and the static approximation for the Glauber gauge boson propagator $D_{\mu \nu}(l) \rightarrow-g_{+-} / l_{\perp}^{2}$. Let us first briefly discuss the corresponding leading-power contribution. In this case the $\mathcal{O}(1 / \sqrt{s})$ terms in the eikonal propagators can be neglected and each gauge boson exchange

\footnotetext{
${ }^{2}$ In the nonrelativistic limit the static Coulomb potential is three-dimensional while in Regge limit it results from the exchange of the Glauber gluons [19] propagating in the two-dimensional transversal space.
} 
is described by the leading term of eq. (2.4) with the corresponding momentum transfer. Then the planar box diagram figure 1 (a) is given by the amplitude $\mathcal{M}_{v}$ with a factor

$$
\frac{\alpha}{\pi} \frac{i t}{(2 \pi)^{2}} \int \frac{\mathrm{d}^{2} l_{\perp} \mathrm{d} l_{+} \mathrm{d} l_{-}}{l_{\perp}^{2}(l-q)_{\perp}^{2}\left(l_{-}+i \epsilon\right)\left(l_{+}-i \epsilon\right)},
$$

where $\alpha=g^{2} /(4 \pi)$. The expression for the nonplanar diagram figure 1(b) differs only by the sign of $l_{+}$and in the sum the antifermion propagators add up to $1 /\left(2 l_{+}-i \epsilon\right)-$ c.c. $=$ $i \pi \delta\left(l_{+}\right)$. By symmetrization the fermion propagator can be reduced to $-i \pi \delta\left(l_{-}\right)$in the same way. After trivial integration over the light-cone components the total leading-power contribution is purely imaginary and can be written as $i \phi \mathcal{M}_{v}$ with the Glauber eikonal phase given by an infrared divergent transverse momentum integral

$$
\phi=-\alpha \int \frac{\mathrm{d}^{2} l_{\perp}}{2 \pi} \frac{q_{\perp}^{2}}{l_{\perp}^{2}(l-q)_{\perp}^{2}}=-\alpha \ln \left(\frac{-t}{\mu^{2}}\right),
$$

where $\mu$ is an infrared regulator. The Glauber phase is known to exponentiate so that the all-order leading-power amplitude is given by $e^{i \phi} \mathcal{M}_{v}$ and has no $\ln \tau$ terms at any order of perturbation theory $[1,2] .^{3}$

The calculation of the double-logarithmic one-loop $\mathcal{O}(\tau)$ amplitude can be performed in a similar way. However, in this case the power suppressed term in the eikonal propagators should be kept and for one of the gauge boson exchanges the contact $\mathcal{O}(\tau)$ part of the amplitude eq. (2.4) should be taken. The planar diagram is then proportional to $\mathcal{M}_{v}+\mathcal{M}_{a}$ i.e. contributes only to the same-helicity fermion scattering. The corresponding coefficient is infrared finite and with double-logarithmic accuracy is given by the integral

$$
-\frac{\alpha}{\pi} \frac{i \tau}{(2 \pi)^{2}} \int \frac{\mathrm{d}^{2} l_{\perp} \mathrm{d} l_{+} \mathrm{d} l_{-}}{l_{\perp}^{2}\left(l_{-}+l_{\perp}^{2} / \sqrt{2 s}\right)\left(l_{+}-l_{\perp}^{2} / \sqrt{2 s}\right)}
$$

represented by the effective theory Feynman diagram in figure 1(c). Note that in eq. (3.4) we put $q=0$ and this expression is valid only for $l \gg q$, which is sufficient for the calculation of the double-logarithmic terms. Integration over $l^{+}$in eq. (3.4) can be performed by using Cauchy theorem and taking the residue of the antifermion propagator pole, which gives $l_{+}=l_{\perp}^{2} /(\sqrt{2 s})$ and $l_{-}>0$. Then the remaining integral has double-logarithmic scaling in the interval $l_{\perp}^{2} / \sqrt{s}<l_{-}<\sqrt{s}$ and $q_{\perp}^{2}<l_{\perp}^{2}<s$. Thus eq. (3.4) with the double-logarithmic accuracy can be rewritten as follows

$$
\frac{\tau \alpha}{2 \pi} \int_{q_{\perp}^{2}}^{s} \frac{\mathrm{d} l_{\perp}^{2}}{l_{\perp}^{2}} \int_{l_{\perp}^{2} / \sqrt{s}}^{\sqrt{s}} \frac{\mathrm{d} l_{-}}{l_{-}}=\tau z \int_{0}^{1} \mathrm{~d} \xi \int_{0}^{\xi} \mathrm{d} \eta=\frac{\tau z}{2},
$$

where we introduce the variables $\xi=\ln \left(l_{\perp}^{2} / s\right) / \ln \tau, \eta=\ln \left(l_{-} / \sqrt{s}\right) / \ln \tau$, and $z=\frac{\alpha}{2 \pi} \ln ^{2} \tau$. As it was discussed before the nonplanar $u$-channel diagram is proportional to $\mathcal{M}_{v}-\mathcal{M}_{a}$ and contributes only to the opposite helicity fermion scattering. In the sum of the planar and nonplanar contributions the axial part cancel and the total one-loop amplitude takes the form

$$
\mathcal{M}^{(1)}=\left(i \phi+\tau z+\mathcal{O}\left(\tau^{2}\right)\right) \mathcal{M}^{(0)},
$$

in agreement with the expansion of the exact result for the chiral amplitudes (see, e.g. [21]).

\footnotetext{
${ }^{3}$ This property is violated by the light-by-light scattering contribution [3, 4] discussed below.
} 


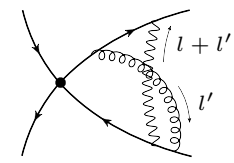

(a)

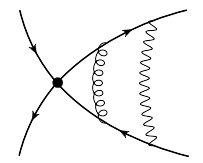

(b)

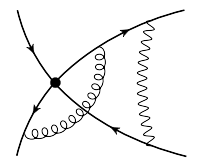

(c)

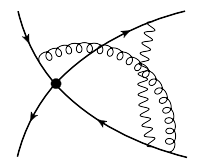

(d)

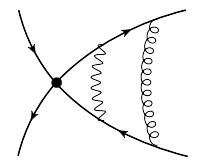

(e)

Figure 2. Two-loop diagrams generating the double-logarithmic power-suppressed corrections. The loopy line corresponds to the leading-power Glauber gauge boson with the momentum $l^{\prime}$. The symmetric diagrams are not shown.

Note that the full effective theory one-loop expression includes also the diagrams with a local $\mathcal{O}\left(g^{2} \tau\right)$ seagull vertex quadratic in the gauge field such as in figure $1(\mathrm{~d})$, which accounts for the off-shell virtual (anti)fermion contribution. These diagrams however do not have double-logarithmic scaling. For example, the diagram in figure $1(\mathrm{~d})$ is ultraviolet finite and depends only on the initial fermion momentum $p_{1}$ and the momentum transfer $q$. Since $p_{1}^{2}=0$ it is a function of two scalar products $\left(p_{1} q\right)$ and $q^{2}$ which are both of order $\mathcal{O}(\tau)$. Hence it cannot generate a large logarithm of the scale ratio, which can be verified by explicit evaluation.

\section{Resummation of subleading-power double logarithms}

In the near-forward scattering the charged particles almost do not accelerate and the usual double-logarithmic corrections associated with the soft gauge boson emission are suppressed [20]. Thus the higher-order double-logarithmic corrections are due to multiple leading-power Glauber gauge boson exchanges which produce infrared-finite terms of the form $\left(\alpha \ln ^{2} \tau\right)^{n}$. To determine the factorization structure of these corrections let us consider the two-loop power-suppressed contribution obtained by dressing the one-loop diagrams with a Glauber gauge boson of the momentum $l^{\prime}$. Typical two-loop effective theory diagrams are presented in figure 2. The corresponding expression can be directly obtained by expanding the full theory diagrams in $l$ and $l^{\prime}$ and keeping only the power suppressed terms linear in $l_{ \pm} \sim \tau$ and quadratic in $l_{\perp} \sim \tau^{1 / 2}$ which give rise to a single insertion of the effective contact interaction. The two remaining Glauber propagators bring the factor $l_{\perp}^{\prime-2}\left(l+l^{\prime}\right)_{\perp}^{-2}$. For $l_{\perp}^{2}<l_{\perp}^{2}$ one can neglect the momentum $l$ in the propagator carrying both loop momenta and the integration over $l_{\perp}$ is nonlogarithmic. Hence in the logarithmic region $l_{\perp}^{2}<l_{\perp}^{2}$ and $l^{\prime}$ should be neglected in the propagator carrying both loop momenta. Thus the integration over $l_{\perp}^{\prime}$ factorizes and one can use the standard properties of the eikonal approximation to study the integral over the light cone components $l_{ \pm}^{\prime}$. Then in the sum of all the diagrams one can separate the part proportional to the product $\delta\left(l_{-}^{\prime}\right) \delta\left(l_{+}^{\prime}\right)$. As for the leading-power contribution it gives the infrared-divergent logarithmic Glauber phase eq. (3.3), which factors out with respect to the one-loop $\mathcal{O}(\tau)$ amplitude. From the expansion of the full theory amplitude we find that the effective theory diagrams with the "soft" Glauber gauge boson coupled to the virtual fermion line have a different multiplicity compared to the diagrams where it couples to the external fermion lines. As a result after separating the Glauber phase the remaining contribution reduces to 
the effective theory diagrams with the characteristic structure shown in figure 2 (a) -2 (c). In the sum of these diagrams with all possible permutations of the gauge boson vertex along the eikonal antifermion line the dependence on the light-cone component of its momentum $l^{\prime}$ can be factored out into $\delta\left(l_{+}^{\prime}\right)$. At the same time the Glauber gauge boson is attached only to the virtual fermion line carrying the loop momentum $l$ and the integration over $l_{-}^{\prime}$ remains logarithmic. The logarithmic integration intervals can be easily identified. As it has been shown above for the transversal component we have $q_{\perp}^{2}<l_{\perp}^{\prime 2}<l_{\perp}^{2}<s$. Then the eikonal fermion propagators bring the factor $\left(l_{-}+l_{\perp}^{2} / \sqrt{2 s}\right)^{-1}\left(l_{-}+l_{-}^{\prime}+\left(l+l^{\prime}\right)_{\perp}^{2} / \sqrt{2 s}\right)^{-1}$. Since $\left(l+l^{\prime}\right)_{\perp}^{2} \approx l_{\perp}^{2}$ it has the logarithmic scaling for $l_{\perp}^{2} / \sqrt{s}<l_{-}<l_{-}^{\prime}<\sqrt{s}$. Thus after integrating over $l_{+}^{\prime}$ the second loop results in a factor

$$
\frac{2 \alpha}{\pi} \int_{q_{\perp}^{2}}^{l_{\perp}^{2}} \frac{\mathrm{d} l_{\perp}^{\prime 2}}{l_{\perp}^{\prime 2}} \int_{l_{-}}^{\sqrt{s}} \frac{\mathrm{d} l_{-}^{\prime}}{l_{-}^{\prime}}=4 z \int_{\xi}^{1} \mathrm{~d} \xi^{\prime} \int_{0}^{\eta} \mathrm{d} \eta^{\prime}=4 z \eta(1-\xi) .
$$

It is straightforward to check that in higher orders the factorization structure remains the same. The $(n+1)$-loop double-logarithmic contribution is generated by the diagrams with a single insertion of the effective contact interaction and $n+1$ leading-power Glauber gauge bosons propagators carrying the momenta $l$ and $l_{i}^{\prime}, i=1, \ldots, n$. Logarithmic scaling of the $l_{\perp}$ integral implies $l_{i \perp}^{\prime 2}<l_{\perp}^{2}$ so that the integrals over $l_{i \perp}^{\prime}$ factorize and the problem is reduced to the standard analysis of the eikonal approximation. As in the two-loop case the Glauber phase factorizes and exponentiates and the double-logarithmic contribution is determined by the sum of the diagrams with all the leading-power vertices attached to the virtual fermion line carrying the loop momentum $l$, as in figure 2(a)-2(c). Again the dependence on $l_{i+}^{\prime}$ factors out to $\delta\left(l_{i_{+}}^{\prime}\right)$ and the logarithmic integration intervals are $q_{\perp}^{2}<$ $l_{i \perp}^{\prime 2}<l_{\perp}^{2}<s$ and $l_{\perp}^{2} / \sqrt{s}<l_{-}<l_{1_{-}}^{\prime}<\ldots<l_{n_{-}}^{\prime}<\sqrt{s}$, i.e. the light-cone components are ordered along the eikonal fermion line. The integration over $l_{i}^{\prime}$ then gives $(4 z \eta(1-\xi))^{n} / n$ ! and after summation over $n$ we get an exponential factor $e^{4 z \eta(1-\xi)}$ to be inserted into the remaining loop integral eq. (3.5). Thus in the leading (double) logarithmic approximation the scattering amplitude reads

$$
\mathcal{M}^{L L}=\tau z g(2 z) \mathcal{M}^{(0)}
$$

where

$$
g(z)=2 \int_{0}^{1} \mathrm{~d} \xi \int_{0}^{\xi} \mathrm{d} \eta e^{2 z \eta(1-\xi)}={ }_{2} F_{2}(1,1 ; 3 / 2,2 ; z / 2)
$$

is the generalized hypergeometric function with the Taylor series expansion $g(z)=1+z / 6+$ $z^{2} / 45+\ldots$. The $\mathcal{O}\left(z^{2}\right)$ contribution to eq. (4.2) agrees with the small-angle expansion of the two-loop result for Bhabha scattering [23-25], which is a nontrivial check of our calculation. It is interesting to note that exactly the same function $g(z)$ appears in the analysis of the double-logarithmic asymptotic behavior of the mass-suppressed QED and QCD amplitudes in the Sudakov limit [17]. Though it is fairly easy to notice a similar factorization structure in both cases, such a universality is rather surprising since in the Sudakov limit the double-logarithmic corrections are associated with the exchange of the on-shell rather than Glauber gauge bosons. 


\begin{tabular}{|c|c|c|c|c|}
\hline & i & ii & iii & iv \\
\hline$\gamma$ & $\frac{\alpha}{2 \pi} \ln |s / t|$ & $\left(\frac{2 \alpha}{\pi}\right)^{1 / 2}$ & $1+\frac{11 \pi}{36} \alpha^{2}$ & $1+\frac{4 \ln 2}{\pi} N_{c} \alpha_{s}$ \\
\hline
\end{tabular}

Table 1. The exponent $\gamma$ defined in the text for (i) the subleading-power scattering, eq. (4.5); (ii) electron-to-muon pair forward annihilation amplitude [12]; (iii) QED Regge cut contribution [3, 4]; (iv) BFKL pomeron contribution $[6,7]$.

The function $g(z)$ has the following asymptotic behavior at $z \rightarrow \infty$

$$
g(z) \sim\left(\frac{2 \pi e^{z}}{z^{3}}\right)^{1 / 2} .
$$

Thus in Regge limit we have

$$
\mathcal{M}^{L L} \sim \frac{\pi / \sqrt{2 \alpha}}{\ln |s / t|}\left|\frac{s}{t}\right|^{-1+\frac{\alpha}{2 \pi} \ln |s / t|} \mathcal{M}^{(0)}
$$

which is the main result of this paper. To characterize the asymptotic behavior of the amplitudes at high energy it is convenient to introduce an exponent $\gamma$ so that $\mathcal{M} \sim s^{\gamma}$ for $s \rightarrow \infty$. Since $\mathcal{M}^{(0)} \sim s$, our result eq. (4.5) corresponds to $\gamma=\frac{\alpha}{2 \pi} \ln |s / t|$. The values of $\gamma$ for a number of different amplitudes and gauge groups are collected in table 1 . In particular it includes the results for the leading-power amplitudes corresponding to the contribution of the rightmost singularities in the complex angular momentum plane: the light-by-light scattering induced Regge cut in QED [3, 4] and the BFKL pomeron Regge pole in QCD [6, 7]. The common feature of all these examples is that $\gamma>0$ and the radiative corrections result in the asymptotic enhancement of the amplitudes. At the same time only for the subleading-power scattering the exponent depends on $\tau$, which is characteristic to the double-logarithmic corrections. By contrast the leading-power effects are single-logarithmic and give energy-independent $\gamma$. On the other hand the QED forward annihilation amplitude [12] is power-suppressed and does get the double-logarithmic corrections. However, at $z \rightarrow \infty$ this amplitude becomes an exponential function of $z^{1 / 2}$ rather than $z$ so that the corresponding exponent $\gamma \sim \sqrt{\alpha}$ is nonanalytic in coupling constant but does not depend on $\tau$ and is consistent with the square root branch point in the complex angular momentum plane. Note that in contrast to the result eq. (4.5), all other asymptotic results in table 1 correspond to the resummation of the ladder diagrams in $t$-channel and can be naturally interpreted in terms of the Regge theory.

In the analysis of the double-logarithmic power-suppressed corrections to the total cross section one also has to consider the back-scattering contribution with $u / s \rightarrow 0$. The leading back-scattering amplitude is power-suppressed with respect to the forward scattering and acquires the double-logarithmic virtual corrections in powers of $\alpha \ln ^{2}|s / u|$. However, in the process of the fermion-antifermion back-scattering the electric charges change the direction of motion to the opposite and the double-logarithmic contribution is dominated by the "bremsstrahlung" on-shell gauge boson exchanges, which result in an exponential suppression of the differential cross section in this kinematical region [12, 20]. 
We can apply the above result to describe the abelian part of the power-suppressed contribution in the color-singled channel of quark-antiquark scattering in QCD. In this case in the expression for $\gamma$ one should use $\alpha=\left(\frac{N_{c}^{2}-1}{4 N_{c}^{2}}\right)^{1 / 2} \alpha_{s}$, where $N_{c}=3$ is the number of colors, $\alpha_{s}$ is the strong coupling constant, and the prefactor arises from projecting on the color-singlet configuration (see e.g. [26]). In principle in the color-singlet channel in abelian approximation one should retain only the contribution of even number of gluons i.e. only even powers of $z$ in the Taylor series for $g(z)$. This however does not change the value of $\gamma$ in the asymptotic formula. Though this result clearly does not give a complete account of subleading-power effects in Regge limit of QCD, it can be considered as a power correction to the BFKL pomeron contribution to the elastic parton scattering [27].

In the high-energy limit for a given $\gamma$ the total cross section has the following scaling $\sigma \sim s^{2(\gamma-1)}$. For $\gamma>1$ such an asymptotic behavior violates the Froissart bound [28] and hence the S-matrix unitarity. This constitutes the unitarity problem of the perturbative Regge analysis, which gives $\gamma>1$ both in QED and QCD, cf. table 1. Though a precise mechanism of unitarity restoration is not yet known (see e.g. [29, 30] for a review), our result sets a perturbative bound on the energy scale where the Regge analysis of the leading-power amplitudes can be trusted. Indeed, for $|\ln \tau| \sim 1 / \alpha$ or more precisely for

$$
\left|\frac{s}{t}\right| \approx e^{2 \pi / \alpha}
$$

the formally power-suppressed contribution to the cross section becomes comparable to the leading-power one and the small-angle expansion breaks down. Interestingly this is roughly the scale at which the resummation of the single-logarithmic corrections responsible for the Regge behavior of the leading-power amplitudes becomes mandatory.

Thus our analysis suggests a completely new scenario for the restoration of unitarity. Note that the nonabelian contribution to eq. (4.5) may alter its asymptotic behaviour and should be computed before a definite conclusion is made in the case of QCD. At the same time eq. (4.5) is complete in QED where the unitarity problem is actually more severe due to its purely perturbative character while in the case of strong interaction a poorly controllable nonperturbative long-distance dynamics may play important role (see e.g. [31]).

\section{Acknowledgments}

I would like to thank Kirill Melnikov for discussions and collaboration and Tao Liu for important cross-checks. This work is supported in part by NSERC and Perimeter Institute for Theoretical Physics.

Open Access. This article is distributed under the terms of the Creative Commons Attribution License (CC-BY 4.0), which permits any use, distribution and reproduction in any medium, provided the original author(s) and source are credited. 


\section{References}

[1] H. Cheng and T.T. Wu, Impact factor and exponentiation in high-energy scattering processes, Phys. Rev. 186 (1969) 1611 [INSPIRE].

[2] S.-J. Chang and S.-K. Ma, Multiphoton exchange amplitudes at infinite energy, Phys. Rev. 188 (1969) 2385 [INSPIRE].

[3] H. Cheng and T.T. Wu, Logarithmic factors in the high-energy behavior of quantum electrodynamics, Phys. Rev. D 1 (1970) 2775 [InSPIRE].

[4] G.V. Frolov, V.N. Gribov and L.N. Lipatov, On Regge poles in quantum electrodynamics, Phys. Lett. B 31 (1970) 34 [INSPIRE].

[5] L.N. Lipatov, Reggeization of the Vector Meson and the Vacuum Singularity in Nonabelian Gauge Theories, Sov. J. Nucl. Phys. 23 (1976) 338 [Yad. Fiz. 23 (1976) 642] [InSPIRE].

[6] E.A. Kuraev, L.N. Lipatov and V.S. Fadin, The Pomeranchuk Singularity in Nonabelian Gauge Theories, Sov. Phys. JETP 45 (1977) 199 [Zh. Eksp. Teor. Fiz. 72 (1977) 377] [INSPIRE].

[7] I.I. Balitsky and L.N. Lipatov, The Pomeranchuk Singularity in Quantum Chromodynamics, Sov. J. Nucl. Phys. 28 (1978) 822 [Yad. Fiz. 28 (1978) 1597] [InSPIRE].

[8] P.D.B. Collins, An introduction to Regge theory and high-energy physics, Cambridge Monographs on Mathematical Physics, Cambridge University Press, Cambridge U.K. (2009).

[9] V.S. Fadin and L.N. Lipatov, BFKL Pomeron in the next-to-leading approximation, Phys. Lett. B 429 (1998) 127 [hep-ph/9802290] [INSPIRE].

[10] A. Luna, S. Melville, S.G. Naculich and C.D. White, Next-to-soft corrections to high energy scattering in QCD and gravity, JHEP 01 (2017) 052 [arXiv:1611.02172] [INSPIRE].

[11] R. Brüser, S. Caron-Huot and J.M. Henn, Subleading Regge limit from a soft anomalous dimension, JHEP 04 (2018) 047 [arXiv: 1802.02524] [INSPIRE].

[12] V.G. Gorshkov, V.N. Gribov, L.N. Lipatov and G.V. Frolov, Doubly logarithmic asymptotic behavior in quantum electrodynamics, Sov. J. Nucl. Phys. 6 (1968) 95 [Yad. Fiz. 6 (1967) 129] [INSPIRE].

[13] J. Bartels, L.N. Lipatov and A. Sabio Vera, Double-logarithms in Einstein-Hilbert gravity and supergravity, JHEP 07 (2014) 056 [arXiv: 1208.3423] [INSPIRE].

[14] A.A. Penin, High-Energy Limit of Quantum Electrodynamics beyond Sudakov Approximation, Phys. Lett. B 745 (2015) 69 [Corrigendum ibid. B 751 (2015) 596] [Corrigendum ibid. B 771 (2017) 633] [arXiv:1412.0671] [INSPIRE].

[15] K. Melnikov and A. Penin, On the light quark mass effects in Higgs boson production in gluon fusion, JHEP 05 (2016) 172 [arXiv: 1602.09020] [INSPIRE].

[16] A.A. Penin and N. Zerf, Two-loop Bhabha Scattering at High Energy beyond Leading Power Approximation, Phys. Lett. B 760 (2016) 816 [Erratum ibid. B 771 (2017) 637] [arXiv: 1606.06344] [INSPIRE].

[17] T. Liu and A.A. Penin, High-Energy Limit of QCD beyond the Sudakov Approximation, Phys. Rev. Lett. 119 (2017) 262001 [arXiv:1709.01092] [INSPIRE].

[18] T. Liu and A. Penin, High-Energy Limit of Mass-Suppressed Amplitudes in Gauge Theories, JHEP 11 (2018) 158 [arXiv: 1809.04950] [INSPIRE]. 
[19] R.J. Glauber, High-Energy Collision Theory, in Lectures in Theoretical Physics. Volume I, W.E. Brittin and L.G. Dunham eds., Interscience Publishers Inc., New York U.S.A. (1959).

[20] V.G. Gorshkov, Electrodynamical processes in opposed high-energy particle beams, Usp. Fiz. Nauk 110 (1973) 45 [INSPIRE].

[21] J.H. Kuhn, S. Moch, A.A. Penin and V.A. Smirnov, Next-to-next-to-leading logarithms in four fermion electroweak processes at high-energy, Nucl. Phys. B 616 (2001) 286 [Erratum ibid. B 648 (2003) 455] [hep-ph/0106298] [INSPIRE].

[22] V.V. Sudakov, Vertex parts at very high-energies in quantum electrodynamics, Sov. Phys. JETP 3 (1956) 65 [Zh. Eksp. Teor. Fiz. 30 (1956) 87] [INSPIRE].

[23] Z. Bern, L.J. Dixon and A. Ghinculov, Two loop correction to Bhabha scattering, Phys. Rev. D 63 (2001) 053007 [hep-ph/0010075] [INSPIRE].

[24] A.A. Penin, Two-loop corrections to Bhabha scattering, Phys. Rev. Lett. 95 (2005) 010408 [hep-ph/0501120] [INSPIRE].

[25] A.A. Penin, Two-loop photonic corrections to massive Bhabha scattering, Nucl. Phys. B 734 (2006) 185 [hep-ph/0508127] [INSPIRE].

[26] T. Liu, K. Melnikov and A.A. Penin, Nonfactorizable QCD Effects in Higgs Boson Production via Vector Boson Fusion, Phys. Rev. Lett. 123 (2019) 122002 [arXiv:1906.10899] [InSPIRE].

[27] A.H. Mueller and W.-K. Tang, High-energy parton-parton elastic scattering in QCD, Phys. Lett. B 284 (1992) 123 [INSPIRE].

[28] M. Froissart, Asymptotic behavior and subtractions in the Mandelstam representation, Phys. Rev. 123 (1961) 1053 [INSPIRE].

[29] H. Cheng and T.T. Wu, Expanding Protons: Scattering at High Energies, The MIT Press, Cambridge U.S.A. (1987).

[30] J.R. Forshaw and D.A. Ross, Quantum Chromodynamics and the Pomeron, Cambridge Lecture Notes in Physics, Cambridge University Press, Cambridge U.K. (1997).

[31] A.H. Mueller and J.-w. Qiu, Gluon Recombination and Shadowing at Small Values of $x$, Nucl. Phys. B 268 (1986) 427 [inSPIRE]. 